\title{
PROPIEDADES PSICOMÉTRICAS DE LA ESCALA DE MIEDO A LA EVALUACIÓN NEGATIVA VERSIÓN BREVE (BFNE) EN MUESTRA CLÍNICA
}

\author{
María J. Gallego ${ }^{1}$, Cristina Botella ${ }^{1,3}$, Soledad Quero ${ }^{1,3}$, Rosa M. Baños ${ }^{2,3}$ Y \\ AZUCENA GARCÍA-PALACIOS ${ }^{1,3}$ \\ ${ }^{1}$ Facultad de Psicología, Universidad Jaime I, Castellón \\ ${ }^{2}$ Facultad de Psicología, Universidad de Valencia \\ ${ }^{3}$ CIBER Fisiopatología Obesidad y Nutrición (CB06/03), Instituto de Salud Carlos III, España.
}

\begin{abstract}
Resumen: La Escala de Miedo a la Evaluación Negativa versión breve (BFNE) fue creada por Leary (1983) a partir de la Escala de Miedo a la Evaluación Negativa (FNE) (Watson y Friend, 1969). El BFNE está formado por 12 ítems con un formato de respuesta tipo Likert de 5 puntos y mide el grado en el que una persona teme ser evaluado negativamente por los demás. Esta escala fue aplicada a una muestra de 122 participantes diagnosticados de fobia social según criterios DSM-IV-TR (2000). En esta muestra, el BFNE obtuvo una estructura bifactorial y la naturaleza de estos factores fue similar a los factores posteriormente informados por Rodebaugh et al. (2004) y Weeks et al. (2005). El BFNE también mostró una consistencia interna alta para ambas subescalas. En cuanto a la validez concurrente obtuvo una correlación significativa con medidas de fobia social, ansiedad rasgo y depresión. Además, el BFNE fue capaz de discriminar entre los diferentes subtipos de fobia social (Heimberg et al., 1993).
\end{abstract}

Palabras clave: Evaluación, fobia social, ansiedad social, miedo a la evaluación negativa.

\section{Psychometric properties of the Brief version of the Fear of Negative Evaluation Scale (BFNE)} in a clinical sample

\begin{abstract}
The Brief version of the Fear of Negative Evaluation Scale (BFNE) was built by Leary (1983a) from the Fear of Negative Evaluation Scale (FNE) (Watson y Friend, 1969). The BFNE consists of 12 items scored on a five-point Likert scale and it assesses the degree to which people fear being evaluated negatively by others. This scale was applied to a sample of 122 participants with a diagnosis of social phobia (DSM-IV, 2000). In this sample the BFNE exhibited a 2-factor structure and the nature of the factors was similar to the factors reported by Rodebaugh et al. (2004) and Weeks et al. (2005). The BFNE showed a high internal consistency for both subescales. With regard to concurrent validity the scale obtained a significant correlation with social phobia, trait anxiety and depression measures. Moreover, the BFNE was able to differentiate among social phobia subtypes (Heimberg et al., 1983).
\end{abstract}

Keywords: Assessment, social phobia, social anxiety, fear of negative evaluation.

\section{INTRODUCCIÓN}

La fobia social se suele definir como «un temor acusado y persistente por una o más situaciones sociales o actuaciones en público en

Recibido: 3 septiembre 2007; aceptado 22 octubre 2007.

Correspondencia: M. J. Gallego Pitarch, Universidad Jaime I, Departamento de Psicología Básica, Clínica y Psicobiología, Avda. Vicent Sos Baynat s/n, 12071 Castellón, España. Correo-e: gallegom@sg.uji.es las que el sujeto se ve expuesto a personas que no pertenecen al ámbito familiar o a la posible evaluación por parte de los demás» (American Psychiatric Association, 2000). Algunos mode-

\footnotetext{
Este estudio ha sido financiado por el Ministerio de Educación y Ciencia (PROYECTOS CONSOLIDER-C): «BIENE-STAR: Nuevas tecnologías de la información y la comunicación: integración y consolidación de su uso en ciencias sociales para mejorar la salud, la calidad de vida y el bienestar» (SEJ2006-14301/PSIC), y por la Universidad Jaime I Fundació Caixa Castelló (P1 1A2005-06).
} 
los cognitivo comportamentales (Clark y Wells, 1995; Rapee y Heimberg, 1997) proponen que la ansiedad social es, en parte, una respuesta a la evaluación negativa de los demás percibida por uno mismo. El miedo a la evaluación negativa es definido por Watson y Friend (1969) como una aprensión hacia la evaluación por parte de los demás, el malestar ante las evaluaciones negativas, y expectativas que una persona puede tener acerca de que los otros la evaluarán negativamente. Este constructo es distinto al concepto de ansiedad social, pero ambos están íntimamente relacionados (Weeks et al, 2005). El miedo a la evaluación negativa forma parte del componente cognitivo de la fobia social, éste está conformado por pensamientos irracionales relacionados con ser evaluado desfavorablemente en situaciones sociales. Por otra parte, la ansiedad social se refiere al componente emocional de la fobia social, este componente está formado por las reacciones afectivas ante situaciones sociales (Weeks et al, 2005).

La Escala de Miedo a la Evaluación Negativa (Fear of Negative Evaluation Scale; FNE) fue construida por Watson y Friend en 1969 y desde entonces es la medida más utilizada para averiguar el grado en el que la gente experimenta aprensión cuando prevé ser evaluado negativamente. Así que las personas que puntúen alto en esta escala tenderán a ponerse nerviosos en situaciones en las que puedan ser evaluadas por los demás. El FNE esta formado por 30 ítems que presentan un formato dicotómico de respuesta (verdadero vs. falso). Se han encontrado buenas propiedades psicométricas en varios estudios con muestras de estudiantes universitarios. La consistencia interna obtenida en distintos estudios es excelente $(\alpha=0,94)$ y la fiabilidad test-retest a un mes fue 0,78 (Watson y Friend, 1969). En cuanto a la validez concurrente, el FNE correlacionó significativamente con Taylor's Manifest Anxiety Scale ( $r=$ 0,60) (Taylor, 1953) y la subescala The Social Approval de Jackson's Personality Research Form $(r=0,77)$ (Jackson, 1966) (Watson y Friend, 1969). Corcoran y Fischer (2000) también estudiaron la validez del FNE y vieron como correlacionaba significativamente con medidas de sintomatología ansiosa y varias medidas de personalidad (por ejemplo, aprobación social y locus de control). Autores como Durm y Glaze (2001) obtuvieron una correlación negativa entre el FNE y la aceptación de uno mismo. Algunos estudios han comparado los individuos que puntúan bajo en el FNE versus los que puntúan alto para evaluar la validez predictiva y de criterio de esta escala. Las personas que puntúan alto en el FNE evitan comparaciones sociales potencialmente amenazantes (Friend y Gilbert, 1973), se sienten peor cuando los evalúan negativamente (Smith y Sarason, 1975), experimentan más ansiedad en situaciones de evaluación (Watson y Friend, 1969), están más preocupados por intentar causar buena impresión a los demás (Leary, 1983b) e identifican sesgadamente las expresiones faciales de los demás como negativas (Winton, Clark y Edelmann, 1995).

Nuestro equipo de investigación llevo a cabo un estudio de validación del FNE en población española no clínica y clínica en el que se obtuvo una consistencia interna moderada para ambas muestras (clínicos $\alpha=0,34$; no clínicos $\alpha=0,49$ ), la estructura factorial resultante fue bidimensional y mostró una buena validez concurrente, ya que correlacionó de forma significativa con medidas de ansiedad social, ansiedad rasgo y depresión (Villa, 1999).

García-López, Olivares, Hidalgo, Beidel y Turner (2001) evaluaron las propiedades psicométricas del FNE en una muestra de adolescentes españoles formada por 202 fóbicos sociales y 101 personas no clínicas. En este estudio el FNE mostró una fiabilidad test-retest alta $(r=$ 0,75 ) y una buena validez de constructo. Por lo que respecta a la validez concurrente el FNE correlacionó significativamente con otras medidas de fobia social. Además estos autores vieron como el FNE puede discriminar entre personas con fobia social circunscrita, fobia social generalizada y personas sin un diagnóstico de fobia social.

Las propiedades psicométricas del FNE también se han investigado en muestras puramente clínicas. Oei, Kenna y Evans (1991) en una muestra de personas con distintos trastornos de ansiedad obtuvieron una consistencia interna alta $(\alpha=0,94)$. Por otra parte, en varios estudios con muestras de fóbicos sociales se ha vis- 
to cómo el FNE correlacionaba significativamente con medidas de ansiedad, depresión y malestar general (Cox, Swinson y Direngeld, 1998; Turner, McCanna y Beidel, 1987). En un estudio de miedo a hablar en público (Rapee y Lim, 1992), el FNE fue el predictor de discrepancias entre la valoración de la ejecución realizada por el participante y por el observador, siendo el participante el que valoró su ejecución peor que el observador. Finalmente, destacar que el FNE ha demostrado ser una de las medidas más sensibles al cambio terapéutico después de haber administrado un tratamiento cognitivo-comportamental para personas con fobia social (Cox et al., 1998; Heimberg et al., 1990).

En el estudio de Tuner et al. (1987) el FNE se administró a personas diagnosticadas de diferentes trastornos de ansiedad (fobia social, fobia simple, agorafobia, trastorno de pánico, trastorno de ansiedad generalizada y trastorno obsesivo-compulsivo), los resultados tan solo mostraron diferencias significativas en el FNE entre las personas con fobias simples y las personas con los demás trastornos de ansiedad, obteniendo una puntuación más baja las personas con fobias simples. Como consecuencia de estos resultados Turner et al. (1987) sacaron las siguientes conclusiones: a) el FNE no se debería usar como único instrumento en los estudios de fobia social, b) el FNE no es una medida relevante en fobia social, y c) este cuestionario es un indicador de malestar general más que de fobia social. Aunque hemos de destacar una debilidad de este estudio, y es que Turner et al. nos informan del $\mathrm{N}$ de la muestra, pero no dicen cuántos participantes sufren cada uno de los trastornos de ansiedad, por lo tanto, no sabemos si cuentan con un $\mathrm{N}$ suficientemente grande en cada uno de los grupos diagnósticos. Además la opinión de Turner et al. en absoluto es compartida por todo el mundo, por ejemplo, Heimberg, Hope, Rapee y Bruch (1988) presentaron estudios que demuestran que el FNE tiene un papel muy importante en la evaluación de la fobia social, y está es la opinión más generalizada.

No obstante, el FNE tiene algunos inconvenientes. Leary (1983a) apuntó que la queja principal de los usuarios del FNE era su longitud, aunque el total de ítems del cuestionario es modesto comparado con otras medidas psicológicas, tiene muchos ítems con contenido redundante. A diferencia de lo que apuntaron Cox et al. (1998) y Heimberg et al. (1990), autores como Leary (1983a) y Saluck et al. (2000) observaron que el formato de respuesta verdadero-falso hacía que el FNE no fuera una medida sensible al cambio terapéutico (Leary, 1983a; Saluck et al., 2000), en nuestro país el profesor Echeburúa (1995) también destaca el carácter dicotómico de esta escala como una de sus debilidades. En este sentido, Nuevo (2005) defiende la concepción dimensional a la hora de evaluar los trastornos mentales frente a la dicotómica. Por otra parte, en opinión de Heimberg (1994) el FNE confunde la evaluación del componente cognitivo y el emocional, en concreto, en el ítem 3 «Me pongo tenso y nervioso si sé que alguien me está analizando/ evaluando» los términos tenso y nervioso hacen referencia a manifestaciones corporales. Sin embargo, hemos de destacar que lo que ocurre en la mayor parte de instrumentos cuando están evaluando ansiedad social evalúan los aspectos cognitivos, fisiológicos y comportamentales de la ansiedad.

Leary (1983a) construyó un instrumento que pudiera subsanar las deficiencias que hemos apuntado y para ello seleccionó 12 ítems de la escala original que correlacionaron al menos 0,50 con la escala total. Esta escala se llamó Escala de Miedo a la Evaluación Negativa version breve (Brief version of the Fear of Negative Evaluation Scale; BFNE), en esta versión reducida el formato de respuesta utilizado fue tipo Likert con 5 alternativas de respuesta, en la que 1 es «nada característico en mí» y 5 es «extremadamente característico en mí», a diferencia de la escala original que tenía un formato de respuesta verdadero-falso. Rodebaugh et al. (2004) y Weeks et al. (2005) pasaron la escala a estudiantes universitarios y a muestra clínica diagnosticada de fobia social y vieron como la estructura de este cuestionario era bifactorial, por una parte, se encontraban los ítems directos (BFNE-S) y, por otra, los ítems inversos (BFNE-R), resultados similares obtuvo Villa (1999) en población clínica y no clínica española. Rodebaugh et al. (2004) vieron cómo la correlación entre ambos factores era 
baja $(r=0,27)$, así que dedujeron que cada factor podía estar midiendo constructos diferentes. Collins, Westra, Dozois y Stewart (2005) convirtieron los ítems inversos del BFNE en directos atendiendo a las críticas que señalaban que los ítems inversos eran confusos y disminuían la validez de la escala (Marsh, 1996; Rodebaugh et al., 2004). La escala resultante se administró a población no clínica y a población clínica, fóbicos sociales y personas con trastorno de pánico, y se obtuvo una estructura factorial unidimensional.

El BFNE demostró una buena consistencia interna $(\alpha=0,90-0,91)$ y una fiabilidad testretest a 4 semanas alta $(r=0,75)$ en muestras de universitarios (Leary, 1983b; Miller, 1995). Weeks et al. (2005) obtuvieron para población no clínica una consistencia interna moderada para el BFNE y el BFNE-R $(\alpha=0,67)$, y alta para el BFNE-S $(\alpha=0,90)$. En cambio, en una muestra de fóbicos sociales la consistencia interna fue alta para la escala total $(\alpha=0,89)$ y ambas subescalas (BFNE-S $\alpha=0,92$ y BFNE$\mathrm{R} \alpha=0,85)$. En nuestro país Villa (1999) administró esta escala a población no clínica ( $\alpha=$ $0,65)$ y a población clínica $(\alpha=0,68)$, obteniendo en ambos casos una consistencia interna alta. Por lo que respecta a la escala transformada en ítems directos Collins et al. (2005) la administraron a una muestra clínica formada por fóbicos sociales y personas con trastorno de pánico, la fiabilidad inter-ítem fue alta $(\alpha=0,97)$ y la fiabilidad test-retest también $(r=$ $0,94)$.

Con respecto a su validez convergente, los estudios llevados a cabo en muestras de universitarios indicaron una correlación alta entre el BFNE y el FNE $(r=0,96)$ (Leary, 1983a; Miller, 1995) y moderada con otras medidas de fobia social (Leary, 1983a); Social Avoidance and Distress (SAD) - Anxiety subescale $(r=$ $0,35)$, SAD - Avoidance subescale $(\mathrm{r}=0,19)$ (Watson y Friend, 1969), y Interaction Anxiousness Scale $(r=0,32)$ (IAS; Leary, 1983c). Los estudios llevados a cabo en población clínica (Weeks et al., 2005) también muestran una correlación moderada entre el BFNE y otras medidas de fobia social: Liebowitch Social Anxiety Scale $(r=0,56)$ (LSAS; Liebowitz, 1987), Social Interaction Anxiety Scale ( $r=$
0,38) (SIAS; Mattick y Clarke, 1998), Social Phobia Scale $(r=0,35)$ (SPS; Mattick y Clarke, 1998) y Fear Questionnaire - Social Phobia subescale $(r=0,35)(\mathrm{FQ}-\mathrm{S}$; Marks y Matthews, 1979). Con respecto a la correlación de las subescalas del BFNE con medidas de fobia social Weeks et al. (2005) obtuvieron correlaciones moderadas entre el BFNE-S y las siguientes medidas: LSAS $(r=0,59)$, SIAS $(r$ $=0,46)$, SPS $(r=0,40)$ y FQ-S $(r=0,40)$. Sin embargo, el BFNE-R tan solo correlacionó con el LSAS $(r=0,26)$. Por otra parte, Collins et al. (2005) también encontraron una correlación moderada entre el FQ-S y el BFNE $(r=0,56)$. En población española Villa (1999) obtuvo correlaciones significativas entre la subescala BFNE-S y la subescala de fobia social del Social Phobia and Anxiety Inventory $(r=0,57)$ (SPAI; Turner, Beidel, Dancu y Stanley, 1989), y entre el BFNE-S y el SAD $(r=0,32)$.

El BFNE también ha mostrado relaciones significativas con otros constructos psicológicos en población clínica (Weeks et al., 2005) tales como sensibilidad a la ansiedad $(r=0,27)$ (Anxiety Sensivity Index, ASI; Reiss, Petersson, Gursky y McNally, 1986), preocupación excesiva $(r=0,51)$ (Penn State Worry Questionnaire, PSWQ; Meyer, Miller, Metzger y Borkovec, 1990) y depresión $(r=0,31)$ (Beck Depression Inventory, BDI; Beck, Rush, Shaw y Emery, 1979). Collins et al. (2005) también obtuvo una correlación moderada $(r=0,32)$ con el Beck Depression Inventory-II (BDI-II; Beck, Steer y Brown, 1996). En una muestra española compuesta por fóbicos sociales y población no clínica (Villa, 1999) el BFNE correlacionó significativamente con medidas de ansiedad general $(r=0,54)$ y autoestima $(r=-0,52)($ Rosenberg Self-Esteem Scale, RSES; Rosenberg, 1965).

Collins et al. (2005) apoyaron la validez discriminante del BFNE, ya que las personas con fobia social puntuaron significativamente más alto que las personas con trastorno de pánico y la población no clínica. Por otra parte, Weeks et al. (2005) vieron como las correlaciones del BFNE con medidas de sensibilidad a la ansiedad (ASI; Reiss et al., 1986) y medidas de depresión (BDI; Beck et al., 1979) fueron bajas, demostrando la validez discriminante del instrumento, sin embargo, el BFNE obtuvo una 
correlación elevada con una medida de preocupación excesiva (PSWQ; Meyer et al., 1990).

La validez de criterio del BFNE fue medida por Leary (1983a), el criterio utilizado se basó en una tarea en la que los participantes tenían que llevar una conversación durante seis minutos con otro participante del estudio. El BFNE correlacionó $r=0,57$ con el grado en el que los participantes se preocupaban por la evaluación negativa del otro, en cambio correlacionó $r=$ 0,31 con el grado en el que los participantes creían que lo estaban haciendo bien. Finalmente, el BFNE ha demostrado ser sensible a los cambios terapéuticos en muestras de fóbicos sociales a los que se administró un tratamiento cognitivo-comportamental para la fobia social (Collins et al., 2005; Weeks et al., 2005) y en personas con trastorno de pánico después de un tratamiento cognitivo-comportamental (Collins et al., 2005).

El objetivo principal de este trabajo es llevar a cabo la validación y adaptación en población clínica española diagnosticada de fobia social del BFNE elaborado por Leary (1983a). El objetivo general se concreta en los siguientes puntos: 1) Analizar la estructura factorial de este cuestionario en población clínica española; 2) Analizar la consistencia interna; 3) Comprobar la validez convergente de esta escala con otras medidas que también se emplean en la evaluación de la fobia social; 4) Comprobar la relación del BFNE con otros constructos psicológicos; y 5) Analizar si existen diferencias en el BFNE en función del diagnóstico según la clasificación de Heimberg et al. (1993).

\section{MÉTODO}

\section{Participantes}

La muestra estaba formada por 122 participantes que formaron parte de un estudio llevado a cabo en el Servicio de Asistencia Psicológica de la Universidad Jaume I sobre el miedo a hablar en público. En este estudio se utilizaron los siguientes criterios de inclusión: a) cumplir criterios DSM-IV para la fobia social, b) duración del trastorno como mínimo de un año, y c) edad entre 18 y 60 años. Y los criterios de exclusión fueron: a) alcoholismo, b) adicción a drogas, c) depresión mayor, d) psicosis, y e) retraso mental.

La mayoría de participantes fueron mujeres $(\mathrm{n}=103)$ y 19 varones. La media de edad fue $24,14(5,34)$, siendo la edad mínima 17 y la máxima 48. Por lo que se refiere al estado civil la mayoría eran solteros $(n=111), 10$ casados y 1 divorciado. En cuanto al nivel de estudios una persona había cursado estudios primarios, 2 estudios secundarios y 119 tenían o estaban cursando estudios superiores. Respecto al diagnóstico atendiendo a la clasificación de Heimberg et al. (1993), 54 participantes cumplieron criterios para la fobia social específica, 44 para la fobia social no generalizada y 24 para la fobia social generalizada.

\section{Entrevistas e instrumentos}

Entrevista de admisión (Botella, 1988). Es una entrevista que se utiliza de forma rutinaria en el Servicio de Asistencia Psicológica y fue diseñada por nuestro grupo de investigación. Esta entrevista es útil para tener una primera aproximación al diagnóstico y permite ver si existen indicios sobre la presencia de MHP en los participantes. Además la entrevista permite rastrear si los participantes cumplen los criterios de inclusión y exclusión anteriormente nombrados.

Entrevista estructurada ADIS-IV (Brown, DiNardo y Barlow, 1994). Entrevista para el diagnóstico de los trastornos de ansiedad siguiendo los criterios del DSM-IV (APA, 1994). Esta entrevista incluye una serie de preguntas que rastrean los criterios diagnósticos y una serie de situaciones sociales respecto a las que el participante ha de indicar el grado de temor y de evitación que presenta en la actualidad. También se analizan los episodios actuales y pasados de ansiedad social, la etiología, la interferencia con el funcionamiento cotidiano y la posible presencia de ataques de pánico.

Escala de Miedo a la Evaluación Negativa versión Breve [Brief version of the Fear of Negative Evaluation Scale, BFNE] (Leary, 1983a). Esta escala evalúa el aspecto más cognitivo de la ansiedad social y para ello se cen- 
tra en el temor que la persona experimenta ante la posibilidad de ser juzgada negativamente por parte de los demás. La escala está formada por 12 ítems y tiene un formato de respuesta tipo Likert de 5 puntos, en la que 1 es «nada característico en mi» y 5 «extremadamente característico en mi» (véase el Anexo 1). Las propiedades psicométricas de este instrumento ya se han comentado anteriormente.

Escala de Evitación y Malestar Social [Social Avoidance and Distress Scale, SAD] (Watson y Friend, 1969). Se trata de un escala de 28 ítems, con dos posibilidades de respuesta (verdadera vs. falso), referidos a la ansiedad que experimenta la persona en situaciones sociales (14 ítems) y la evitación activa o deseo de evitación de esas situaciones (14 ítems). En cuanto a la fiabilidad esta escala presenta una consistencia interna alta $(\alpha=0,94)$ y una buena estabilidad temporal $(1 \mathrm{mes})(r=0,68)$ (Watson y Friend, 1969).

Cuestionario de Autoeficacia al Intervenir en Público (CAIP; adaptado por Bados, 1986). Nuestro equipo de investigación adaptó este instrumento para esta investigación a partir del cuestionario de «Autoeficacia para hablar en público» (Bados, 1986). Esta medida fue validada en población española y mostró una buena fiabilidad test-restest $(r=0,92)$ (Bados, 1986). El instrumento contiene 6 situaciones que implican intervenir en público y cada una de ellas conlleva distintos grados de dificultad. Este cuestionario mide el grado en que la persona puede afrontar diferentes situaciones relacionadas con hablar en público, utilizando una escala de 1 («claramente no podría realizarlo») a 10 («claramente sí podría realizarlo»).

Autoverbalizaciones durante la situación de hablar en público [Self-Statements During Public Speaking, SSPS] (Hofmann y DiBartolo, 2000). Es un cuestionario de 10 ítems basado en el SISST (Social Interaction and Self-Statement Test) de Glass et al. (1982) y mide las autoafirmaciones de la persona y el grado de malestar experimentado mientras habla en público. Su formato de respuesta es tipo Likert que va de 0 («en absoluto de acuerdo») a 5 («totalmente de acuerdo») y presenta dos subescalas: la subescala de autoverbalizaciones positivas (SSPS-P) y la subescala de autoverba- lizaciones negativas (SSPS-N). Las dos subescalas del SSPS mostraron una buena consistencia interna, tanto para la subescala SSPS-P $(\cdot=$ $0,80)$ como en la subescala SSPS-N $(\cdot=0,86)$, y una buena fiabilidad test-retest a los 3 meses (SSPS-P $r=0,78$; SSPS-N $r=0,80$ ) (Hofmann y DiBartolo, 2000).

Cuestionario de Confianza para Hablar en Público [Personal Report of Confidence as Speaker, PRCS] (Paul, 1966). Adaptado a población española por Bados (1986). Esta escala fue diseñada originalmente por Gilkinson (1942) y posteriormente modificada por Paul (1966). Los 101 ítems originales se redujeron en la segunda versión a 30 ítems con un formato de verdadero-falso. En España, Bados (1986) sustituyó el formato de respuesta verdadera-falso por una escala de 6 puntos, de 1 («completamente de acuerdo») a 6 («completamente en desacuerdo»). Los resultados se interpretan en el sentido de a mayor puntuación, más miedo a hablar en público. Méndez, Inglés y Hidalgo (1999) estudiaron las propiedades psicométricas del PRCS (Bados, 1986) en población adolescente española y obtuvieron una estructura unifactorial y un coeficiente $\cdot$ de Cronbach alto $(0,91)$.

Inventario de ansiedad estado-rasgo (STAI; Spielberger, Gorsuch y Lushene, 1983). Adaptado por TEA (1988) para población española. Este cuestionario consta de dos escalas, que están formadas por 20 ítems cada una. En la escala de ansiedad-rasgo, los participantes tienen que describir cómo se sienten generalmente, y en la escala ansiedad-estado, cómo se sienten en el momento de contestar. Las categorías de respuesta en la escala ansiedad-rasgo son: casi nunca, a veces, a menudo y casi siempre. En la escala de ansiedad-estado las categorías son: nada, algo, bastante y mucho. El cuestionario permite obtener puntuaciones para las dos escalas. El coeficiente de fiabilidad test-retest en el caso de la escala ansiedad-estado es bajo $(0,40)$, como es deseable, mientras que para la ansiedad-rasgo es de 0,81 .

Inventario de Depresión de Beck (BDI-13; Beck, Rial y Rickels, 1974). Adaptado por Conde y Franch (1984). Beck et al. (1974) realizaron una versión corta de 13 ítems entresacados de los 21 ítems originales (Beck, Ward, Men- 
delson, Mock y Ergbaugh, 1961). En esta versión, al igual que en la anterior, los ítems tenían cuatro alternativas de respuesta ordenadas de menor a mayor intensidad. La persona debe señalar cual de las afirmaciones describe mejor sus sentimientos actualmente. Se puntúa de cero a tres cada uno de los ítems y la puntuación total es el sumatorio de todas las puntuaciones. Los puntos de corte son los siguientes, 0-4: depresión ausente o mínima; 5-7: depresión leve; 5-15: depresión moderada; 16 o más: depresión grave. Por lo que respecta a la correlación entre la forma corta y la forma larga es $r=0,96$.

\section{Procedimiento}

En el presente estudio utilizamos los 12 ítems que conforman el BFNE de la escala FNE traducida al español y validada en población española (García-López et al., 2001; Villa et al., 1998). Desde el Servicio de Asistencia Psicológica se pusieron anuncios utilizando el e-mail y carteles en centros de enseñanza y de salud de Valencia y Castellón. Estos carteles informaban acerca de la puesta en marcha de un programa de tratamiento gratuito para el miedo a hablar en público. Se llevaron a cabo las entrevistas oportunas a las personas que solicitaron ayuda utilizando la entrevista de admisión y, posteriormente, si cumplían los criterios de inclusión y exclusión se establecía el diagnóstico mediante la entrevista ADIS-IV (Brown et al., 1994). Los diagnósticos fueron efectuados por una psicóloga experta en psicología clínica de acuerdo con los datos de la entrevista y siguiendo los criterios Heimberg et al. (1993). De acuerdo con estos autores, la psicóloga diagnosticaba fobia social generalizada cuando la ansiedad estaba presente en la mayoría de situaciones sociales. Diagnosticaba fobia social no generalizada cuando las personas cumplían las siguientes condiciones: pueden tener o no miedos específicos de ejecución; experimentan una ansiedad clínicamente significativa en situaciones de interacción social; pero, no experimentan una ansiedad significativa en, al menos, un área de su funcionamiento social. Finalmente, se diagnosticaba fobia social específica, cuando la persona presenta ansiedad en un número limitado de situaciones muy concretas.

Las personas que cumplían criterios de fobia social y quisieron firmar el consentimiento informado (hoja en la que después de recibir información sobre el estudio la persona acepta voluntariamente participar en éste y también que los datos que se deriven del mismo puedan ser utilizados para investigación) cumplimentaron el resto de instrumentos comentados con anterioridad e iniciaron un programa de tratamiento cognitivo-comportamental para la fobia social (Gallego, 2007).

\section{RESULTADOS}

\section{Estructura factorial del BFNE}

En primer lugar, se realizaron las pruebas estadísticas para determinar si los datos eran susceptibles de someterse a un análisis factorial. En el test de Kaiser-Meyer-Olkin (KMO) se obtuvo un índice de 0,88 y en la prueba de esferidad de Barlett $\chi^{2}(66)=729,75, p<0,001$. Dado que el índice de la prueba KMO fue mayor que 0,60 y que la prueba de Barlett resultó significativa, se puede concluir que es adecuado realizar un análisis factorial con los datos.

Por tanto, se realizó un primer análisis factorial exploratorio utilizando el método de extracción de componentes principales con rotación varimax. El análisis factorial reveló dos factores con autovalores mayores de uno. También el test de Cattell (scree test, 1966) para las magnitudes de los autovalores sugirió que la solución más parsimoniosa de los datos es la que propone dos factores. Estos dos factores explicaban el $61,44 \%$ de la varianza total, el factor 1 con un valor propio de 5,47 explicaría el $47,54 \%$ de la varianza y el factor 2 con un valor propio de 3,20 explicaría el $13,90 \%$ de la varianza.

El análisis exploratorio nos sugirió una estructura bifactorial. Estos datos, junto con los resultados obtenidos por Rodebaugh et al. (2004), Weeks et al. (2005) y Villa (1999), y el hecho de que las dos subescalas estén altamente correlacionadas $(r=0,45)$, nos llevaron a la 
realización de un segundo análisis factorial con rotación oblimin que confirmó esta estructura. Así que la estructura factorial resultante fue bidimensional, un factor coincidió con los ítems directos (BFNE-S) y el otro con los ítems inversos (BFNE-R). La saturación de cada uno de los ítems con el factor al que corresponden la podemos observar en la Tabla 1 y vemos como en todos los casos fue mayor a 0,60. Ambas subescalas resultantes obtuvieron una correlación alta con la puntuación total del BFNE, aunque la subescala BFNE-S $(r=0,92)$ tiene una correlación más elevada que el BFNE-R $(r=0,65)$ con la escala total.

\section{Análisis descriptivo de los ítems}

En cuanto al análisis descriptivo de los ítems el rango de puntuaciones medias dadas a los ítems osciló entre 4,31 y 3,06. Los ítems que puntuaron más alto como podemos ver en la Tabla 2 fueron el 2 y el 10, ambos ítems son inversos y tenemos que tener en cuenta que los ítems están formulados tal y como los leyeron los participantes en el cuestionario, pero la media y la desviación típica corresponden a la puntuación invertida. Por otra parte, los que puntuaron menos fueron el 6 y el 8 (véase la Tabla 2).
Tabla 1. Cargas factoriales y comunalidades para los ítems del BFNE $(n=122)$

\begin{tabular}{|c|c|c|c|}
\hline $\begin{array}{l}\text { Subescala y } \\
\text { n. }{ }^{\circ} \text { de ítem }\end{array}$ & Factor 1 & Factor 2 & Comunalidades \\
\hline \multicolumn{4}{|l|}{ BFNE-S } \\
\hline 1 & 0,76 & $-0,13$ & 0,59 \\
\hline 3 & 0,77 & $-0,22$ & 0,64 \\
\hline 5 & 0,78 & $-0,06$ & 0,61 \\
\hline 6 & 0,76 & $-0,17$ & 0,61 \\
\hline 8 & 0,77 & $-0,16$ & 0,61 \\
\hline 9 & 0,78 & $-0,29$ & 0,70 \\
\hline 11 & 0,74 & $-0,26$ & 0,61 \\
\hline 12 & 0,80 & $-0,17$ & 0,67 \\
\hline \multicolumn{4}{|l|}{ BFNE-R } \\
\hline 2 & 0,43 & 0,64 & 0,59 \\
\hline 4 & 0,64 & 0,53 & 0,69 \\
\hline 7 & 0,43 & 0,65 & 0,60 \\
\hline 10 & 0,46 & 0,52 & 0,47 \\
\hline
\end{tabular}

\section{Consistencia interna}

Para analizar la consistencia interna, se evaluaron las respuestas de la muestra a través del cálculo del coeficiente $\alpha$ de Cronbach tanto para la escala total, como para cada una de las subescalas. El coeficiente $\alpha$ de Cronbach para la escala total fue 0,90 , para la subescala BFNE-S 0,91 y para la subescala BFNE-R

Tabla 2. Análisis descriptivo de los ítems

\begin{tabular}{|c|c|c|c|}
\hline & $M$ & DT & $\begin{array}{c}\text { Correlaciones } \\
\text { corregidas }\end{array}$ \\
\hline $\begin{array}{l}\text { 1. Me preocupa lo que la gente pensará de mí, incluso cuando sé que no tiene } \\
\text { importancia. }\end{array}$ & 3,48 & 1,07 & 0,69 \\
\hline $\begin{array}{l}\text { 2. No me preocupo incluso cuando sé que la gente se está formando una } \\
\text { impresión desfavorable de mí. (I) }\end{array}$ & 4,31 & 0,97 & 0,38 \\
\hline 3. Frecuentemente temo que otras personas se den cuenta de mis limitaciones. & 3,20 & 1,27 & 0,68 \\
\hline 4. Raramente me preocupo de la impresión que estoy causando en los demás. (I) & 4,16 & 0,97 & 0,59 \\
\hline 5. Temo que los demás no me aprueben. & 3,28 & 1,19 & 0,71 \\
\hline 6. Me da miedo que la gente descubra mis defectos. & 3,06 & 1,17 & 0,69 \\
\hline 7. Las opiniones de los demás sobre mí no me molestan. (I) & 4,08 & 0,94 & 0,38 \\
\hline $\begin{array}{l}\text { 8. Cuando estoy hablando con alguien me preocupa lo que pueda estar pensando } \\
\text { de mí. }\end{array}$ & 3,18 & 1,17 & 0,69 \\
\hline 9. Generalmente me preocupo por la impresión que pueda causar. & 3,45 & 1,08 & 0,70 \\
\hline 10. Si sé que alguien me está juzgando, eso tiene poco efecto sobre mí. (I) & 4,18 & 1,10 & 0,40 \\
\hline $\begin{array}{l}\text { 11. A veces pienso que estoy demasiado preocupado por lo que otras personas } \\
\text { piensan de mí. }\end{array}$ & 3,36 & 1,25 & 0,65 \\
\hline 12. A menudo me preocupa decir o hacer cosas equivocadas. & 3,63 & 1,03 & 0,72 \\
\hline
\end{tabular}

Nota: $\mathrm{I}=$ items inversos. 
0,76. El rango de correlaciones de cada uno de los ítems con la puntuación total del BFNE corregida (es decir, la puntuación total sin tener en cuenta el ítem en cuestión) osciló entre 0,72 y 0,38 , siendo los ítems que menor correlación presentaron el 2 y el 7 , y el que mayor correlación presentó el 12 (ver Tabla 2). Por lo que respecta a la subescala BFNE-S la fiabilidad fue alta $\alpha=0,91$. El rango de correlaciones de cada uno de los ítems con la puntuación del BFNE$\mathrm{S}$ corregida osciló entre 0,76 (ítem 9) y 0,69 (ítem 1). En cuanto a la fiabilidad para la subescala BFNE-R el $\alpha$ de Cronbach fue 0,75, por lo que se refiere al rango de correlaciones de cada uno de los ítems con la puntuación del BFNE-R corregida osciló entre 0,65 (ítem 4) y 0,48 (ítem 10).

\section{Datos normativos}

En la Tabla 3 podemos ver las medias y desviaciones típicas obtenidas por la muestra total (participantes con diagnóstico de fobia social) en la escala total del BFNE y en ambas subescalas.

Tabla 3. Medias y desviaciones típicas del BFNE para población clínica

\begin{tabular}{lcc}
\hline & $\begin{array}{c}\text { Varones } \\
\text { Media }(D T)\end{array}$ & $\begin{array}{c}\text { Mujeres } \\
\text { Media }(D T)\end{array}$ \\
\hline BFNE & $41,95(9,20)$ & $43,45(9,23)$ \\
BFNE-S & $27,16(6,74)$ & $26,54(7,41)$ \\
BFNE-R & $16,56(2,85)$ & $16,76(3,07)$ \\
\hline
\end{tabular}

\section{Validez convergente}

Se analizó la validez convergente del BFNE mediante el cálculo del coeficiente de correlación producto-momento de Pearson con otras medidas de fobia social, de depresión y de ansiedad. La correlación más alta fue con el $\operatorname{SAD}(r=0,52)$, aunque el BFNE también correlacionó significativamente con la mayoría de medidas de miedo a hablar en público (CMHP $r=0,35$; SSPS-P $r=-0,35$; SSPS-N $r$ $=0,41 ;$ PRCS $r=0,42)$, a excepción del CAIP $(r=-0,15)$. Por otra parte, correlaciona moderadamente con el BDI $(r=0,43)$ y el STAI-R $(r$ $=0,47)$, sin embargo, la correlación con el STAI-E $(r=0,21)$ fue baja.

Las correlaciones de la subescala BFNE-S con las medidas citadas son prácticamente las mismas que las obtenidas por el BFNE. Por otra parte, el BFNE-R no correlacionó significativamente con el SSPS-N, el CAIP y el STAI-E, sin embargo, obtuvo una correlación significativa a un nivel de significación $p<0,05$ con el STAI$\mathrm{R}$, el SAD, el CMHP y el SSPS-P. Las únicas medidas que correlacionaron con el BFNE-R a un nivel de significación de $p<0,001$ fueron el PRCS y el BDI. (Véase la Tabla 4).

\section{Puntuaciones diferenciales en el BFNE en función de la clasificación diagnóstica de Heimberg et al. (1993)}

Se realizó un análisis de varianza tomando como variable dependiente la puntuación del

Tabla 4. Análisis de correlaciones entre el BFNE y otras medidas

\begin{tabular}{lccc}
\hline & BFNE & BFNE-S & BFNE-R \\
\hline Social Avoidance and Distress Scale (SAD) & $0,52^{* *}$ & $0,50^{* *}$ & $0,26^{*}$ \\
Cuestionario de Miedo a Hablar en Público (CMHP) & $0,35^{* *}$ & $0,34^{* *}$ & $0,20^{*}$ \\
Cuestionario de Autoeficacia al Intervenir en Público (CAIP) & $-0,15$ & $-0,13$ & 0,05 \\
Positive Self-Statements During Public Speaking (SSPS-P) & $-0,35^{* *}$ & $-0,36^{* *}$ & $-0,21^{*}$ \\
Negative Self-Statements During Public Speaking (SSPS-N) & $0,41^{* *}$ & $0,45^{* *}$ & 0,14 \\
Personal Report of Confidence as Speaker (PRCS) & $0,42^{* *}$ & $-0,37^{* *}$ & $-0,32^{* *}$ \\
Inventario de Depresión de Beck (BDI) & $0,43^{* *}$ & $0,38^{* *}$ & $0,36^{* *}$ \\
Inventario de ansiedad estado-estado (STAI-E) & $0,21^{*}$ & $0,26^{*}$ & 0,07 \\
Inventario de ansiedad estado-rasgo (STAI-R) & $0,47^{* *}$ & $0,45^{* *}$ & $0,24^{*}$ \\
\hline
\end{tabular}

$* p<0,05 ; * * p<0,001$. 
Tabla 5. Puntuaciones en el BFNE en función de la clasificación de Heimberg et al. (1993)

\begin{tabular}{lccc}
\hline & \multicolumn{1}{c}{ Grupos } & $\mathrm{N}$ & Media $(D T)$ \\
\hline $\begin{array}{l}\text { Diagnóstico } \\
\text { según }\end{array}$ & $\begin{array}{c}\text { Fobia social } \\
\text { específica }\end{array}$ & 53 & $40,34(8,60)$ \\
$\begin{array}{l}\text { Heimberg } \\
\text { et al. } \\
(1993)\end{array}$ & $\begin{array}{c}\text { Fobia social } \\
\text { no generalizada }\end{array}$ & 44 & $43,93(9,54)$ \\
& $\begin{array}{c}\text { Fobia social } \\
\text { generalizada }\end{array}$ & 24 & $48,17(7,91)$ \\
\hline
\end{tabular}

BFNE y como factor entre-grupos el diagnóstico según la clasificación de Heimberg et al. (1993), i.e., fobia social generalizada, fobia social no generalizada, y fobia social específica. Se obtuvieron diferencias significativas entre el grupo con fobia social específica, el grupo con fobia social no generalizada y el grupo de fobia social generalizada $(F(2,118)=$ $6,73, p<0,005)$ en la puntuación del BFNE. El análisis post-hoc (HSD Tukey) reveló que el grupo diagnosticado de fobia social específica era significativamente distinto al grupo de fobia social generalizada (límite inferior $=-12,98$, límite superior $=-2,67 ; p<0,005)$, sin embargo, no se encontraron diferencias significativas entre el grupo con fobia social específica y el de fobia social no generalizada, así como entre el grupo con fobia social no generalizada y el de fobia social generalizada.

\section{DISCUSIÓN}

En los resultados se obtiene una estructura factorial bidimensional al igual que en los estudios de Weeks et al. (2005) y Villa (1999) 1levados a cabo en población española clínica y no clínica, y en el trabajo de Rodebaugh et al. (2004) realizado en población no clínica. Los ítems directos de la escala forman el primer factor (BFNE-S) que explica el 47,54\% de la varianza y los inversos el segundo factor (BFNE-R) que explica el 13,90\% de la varian$\mathrm{za}$, finalmente, entre ambos factores se explica el $61,44 \%$ de la varianza total. Así que más que dos dimensiones de la escala ambos factores parecen formar parte de la metodología de corrección del test. Por lo tanto, consideramos seria importante averiguar en futuras investigaciones si es mejor mantener los ítems inversos o al igual que Collins et al. (2005) plantearlos de forma afirmativa y obtener una estructura unidimensional, nosotros creemos que es mejor la segunda opción ya que lo que se manifiesta en los datos es que quizá resulte más confuso para los participantes utilizar la escala invertida, además si observamos la Tabla 1 vemos que todos los ítems del segundo factor obtienen cargas factoriales superiores a 0,40 en el primer factor, esto nos indica que estos ítems podrían formar parte del primer factor.

Por lo que se refiere al análisis descriptivo de los ítems se puso de manifiesto que a las personas diagnosticadas de fobia social les preocupa mucho que la gente se esté formando una opinión desfavorable de ellos (ítem 2), además si saben que les están juzgando, eso tiene efecto sobre ellos (ítem 10). Por otra parte, los ítems que puntúan más bajo son el 6 y el 8 , por lo tanto, vemos como es moderadamente característico en las personas con fobia social que les dé miedo que la gente descubra sus defectos, así como que se preocupen cuando están hablando con alguien de lo que puedan estar pensando de ellos.

En cuanto a la fiabilidad, el BFNE ha mostrado una consistencia interna excelente, tanto para la subescala BFNE-S $(\alpha=0,91)$ como para la escala total $(\alpha=0,90)$, sin embargo, la subescala BFNE-R $(\alpha=0,76)$ obtuvo una consistencia interna más baja. Al igual que en el estudio de Weeks et al. (2005) en población no clínica y en población clínica la consistencia interna obtenida ha sido mayor en la escala total $y$ en la subescala BFNE-S que en la BFNE-R. Respecto a un estudio anterior llevado a cabo por nuestro equipo de investigación en población española clínica la consistencia interna obtenida en el presente estudio para la escala total ha sido más elevada $(\alpha=0,90)$ que la obtenida en el anterior estudio $(\alpha=0,68)$ (Villa, 1999). Sin embargo, la consistencia interna obtenida en el presente estudio es la misma que la obtenida en el estudio original en muestra anglosajona (Leary, 1983).

Por lo que respecta a los datos normativos obtenidos en este estudio con población clínica para la escala total del BFNE (Varones: $M=$ 
41,95, $D T=9,20$; Mujeres; $M=43,45, D T=$ 9,23 ), vemos como las medias son mucho más altas que las obtenidas en población no clínica española (varones: $M=26,50, D T=4,71$; mujeres; $M=27,61, D T=5,40$ ) (Villa, 1999), lo cual manifiesta la gravedad del grupo de personas con fobia social que conformó nuestra muestra. Nuestros resultados ponen de manifiesto la importancia de utilizar el FNE en fobia social, en contra de lo que opinaba Turner et al. (1987) y a favor de lo que opinaba Heimberg et al. (1988).

En lo referido a la validez convergente de este instrumento en el presente estudio se han comprobado las relaciones significativas entre el BFNE y otras medidas de fobia social, depresión y ansiedad general. La correlación más elevada fue con el SAD, es decir, con una medida de fobia social que evalúa la evitación y el malestar en situaciones sociales, Leary (1983a) en el estudio original también encontró está relación. La única correlación que no resultó significativa fue con el CAIP, es decir, el miedo a la evaluación negativa no está relacionado con la medida en que una persona se siente eficaz a la hora de hablar en público. En cuanto a la correlación obtenida entre el BFNE y el STAI-E fue baja, por lo tanto, no daba muestra de una relación importante entre el miedo a la evaluación negativa y la ansiedad que experimentaban en ese momento. Sin embargo, el BFNE y el STAI-R estaban bastante relacionados, al igual que con el BDI. Así que parece que el miedo a la evaluación negativa está relacionado con ansiedad rasgo y depresión, y no tanto en cómo se siente una persona justo en ese momento. Por lo que se refiere a las medidas de fobia social específica, tipo miedo a hablar en público, estaban relacionadas significativamente con el BFNE a excepción del CAIP, como ya hemos dicho anteriormente. Las relaciones obtenidas para la subescala BFNE$\mathrm{S}$ han sido prácticamente las mismas que las obtenidas en la escala total, en cambio la subescala BFNE-R en general ha obtenido correlaciones más bajas y hemos visto como no está relacionada con las autoverbalizaciones negativas al hablar en público (SSPS-N), la medida en que una personas se siente eficaz para hablar en público (CAIP) y la ansiedad que experi- mentaba en el momento de la evaluación (STAI-E). Finalmente, hemos de destacar que al igual que en otros estudios anteriores (Leary, 1983a; Weeks et al., 2003) el BFNE ha demostrado estar relacionado con otras medidas de fobia social.

$\mathrm{Al}$ igual que se plantearon Belloch, Morillo y García-Soriano (2006) la existencia de diferentes subtipos en el trastorno obsesivo-compulsivo, nosotros nos planteamos la existencia de subtipos en la fobia social. Los resultados obtenidos indicaron diferencias significativas en el BFNE en función de los subtipos de fobia social (Heimberg et al., 1993). Fueron las personas diagnosticadas de fobia social específica las que menos temían a la evaluación negativa, seguidas de las personas con fobia social no generalizada $y$, finalmente, las que puntuaron más fueron las personas con fobia social generalizada. Creemos que este continuo es importante e indica la conveniencia de utilizar este instrumento en el caso de personas con un diagnóstico de fobia social.

De los resultados obtenidos se desprende que el BFNE construido por Leary (1983a) constituye una medida adecuada del miedo a la evaluación negativa en población clínica española diagnosticada de fobia social. Aunque en este punto hemos de destacar que los instrumentos autoadministrados son una fuente más de información que debe utilizarse junto a las entrevistas y el juicio del clínico (Fernández-Montalvo y Echeburúa, 2006) como se ha hecho en el presente estudio.

Finalmente, señalar que el presente estudio cuenta con algunas limitaciones que sería recomendable subsanar en investigaciones posteriores, como la edad de los participantes, se trata de una muestra joven aunque todas ellas son mayores de edad; el género, la mayoría son mujeres; y el nivel de estudios, la mayoría tenían o estaban cursando estudios superiores. En el trabajo de Jorquera, Baños, Perpiñá y Botella (2005) se hizo una retrotraducción, esto sería recomendable para futuras investigaciones del BFNE. También sería conveniente que se volviera a realizar un estudio de características similares en el que todos los ítems estuvieran formulados de forma afirmativa. Además sería interesante realizar estudios comparando los 
datos antes frente a después del tratamiento de la fobia social para averiguar la sensibilidad al cambio terapéutico de la escala.

\section{REFERENCIAS BIBLIOGRÁFICAS}

American Psychiatric Association (APA) (2000). Diagnostic and statistical manual of mental disorder (5. ed.). Washington, DC: APA (Barcelona, Masson, 2000).

American Psychiatric Association (APA) (1994). Diagnostical and Statistical Manual of Mental Disorders (4. ${ }^{\mathrm{a}}$ ed.). Washington, DC: APA (Barcelona, Masson, 1995).

Bados, A. (1986). Análisis de componentes de un tratamiento cognitivo-somático-conductual del miedo a hablar en público. Tesis doctoral no publicada, Universidad de Barcelona.

Beck, A. T., Rial, W. Y., y Richels, K. (1974). Short form of Depression Inventory (Cross-validation). Psychological Reports, 34, 1184-1186.

Beck, A. T., Rush, A. J., Shaw, B. F., y Emery, G. (1979). Cognitive therapy of depression. New York: Guilford Press.

Beck, A. T., Steer, R.A., y Brown, G. K. (1996). Beck Depression Inventory manual (2nd ed.). San Antonio: Psychological Corporation.

Beck, A., Steer, R. A., y Garbin, M. G. (1988). Psychometric propierties of the Beck Depression Inventory: Twenty-five years of evaluation. Clinical Psychology Review, 8, 77-100.

Beck, S., Ward, C., Mendelson, M., Mock, J., y Ergbaugh, J. (1961). An inventory for measuring depression. Archives of General Psychiatry, 4, 561-571.

Belloch, A., Morillo, C., y García-Soriano, G. (2006). Obsession subtypes: relationships with obsessive-compulsive symptoms, dysfunctional beliefs and thought control strategies. Revista de Psicopatología y Psicología Clínica, 11, 65-78.

Botella, C. (1988). Entrevista de admisión para los pacientes que solicitan ayuda psicológica. Manuscrito no publicado.

Brown, T. A., DiNardo, P., y Barlow, D. H. (1994). Anxiety Disorders Interview Schedule for DSM-IV (ADS-IV). Adult and Lifetime version. Clinician Manual. San Antonio: Psychological Corporation.

Cattell, R. B. (1966). The scree test for the number of factors. Multivariate Behavioral Research, 1, 245-276.

Clark, D. M., y Wells, A. (1995). A cognitive model of social phobia. En R. G. Heimberg, M. R. Liebowitz, D.A. Hope y F. R. Schneier, Social Phobia: Diagnosis, Assessment, and treatment (pp. 69-93). Nueva York: Guilford Press.
Collins, K. A., Westra, H. A., Dozois, D. J. A., y Stewart, S. H. (2005). The validity of the brief version of the Fear of Negative Evaluation Scale. Anxiety Disorders, 19, 345-359.

Conde, V., y Franch, J. I. (1984). Escalas de evaluación comportamental para la cuantificación de la sintomatología psicopatológica en los trastornos angustiosos y depresivos. Madrid: Laboratorios Upjohn.

Corcovan, K., y Fischer, J. (2000). Measures for clinical practice: a sourcebook. Vol. 2. Adults (3rd ed.). New York: The Free Press.

Cox, B. J., Swinson, R. P., y Direngeld, D. M. (1998). A comparison of social phobia outcome measures in cognitive-behaviorual group therapy. Behavior Modification, 22, 285-297.

Durm, M. W., y Glaze, P. E. (2001). Construct validity for self-acceptance and fear of negative evaluation. Psychological Reports, 89, 386

Echeburúa, E. (1995). Manual práctico de evaluación y tratamiento de la fobia social. Barcelona: Martínez Roca.

Fernández-Montalvo, J., y Echeburúa, E. (2006). Uso y abuso de los autoinformes en la evaluación de los trastornos de personalidad. Revista de Psicopatología y Psicología Clínica, 11, 1-12.

Friend, R., y Gilbert, J. (1973). Threat and fear of negative evaluation as determinants of locus of social comparison. Journal of Personality, 41, 328-340.

Gallego, M. J. (2007). Un estudio controlado que compara un tratamiento autoadministrado vía Internet para el miedo a hablar en público vs. el mismo tratamiento administrado por el terapeuta. http://www.tesisenxarxa.net/TDX-0606107-121943/ : Servidor de Tesis Doctorals en Xarxa.

García-López, L. J., Olivares, J., Hidalgo, M. D., Beidel, D. C., y Turner, S. M. (2001). Psychometric Propierties of the Social Anxiety Scale for Adolescents, the Fear of Negative Evaluation Scale, and the Social Avoidance and Distress Scale in an Adolescent Spanish-Speaking Simple. Journal of Psychopathology and Behavioral Assessment, 33, 51-59.

Gilkinson, H. (1942). Social fears as reported by students in collage speech classes. Speech Monoghraphy, 9, 141-160.

Glass, C. R., Merluzzi, T. V., Biever, J. L., y Larsen, K. H. (1982). Cognitive assessment of social phobia: Development and validation of a self-statement questionnaire. Cognitive Therapy and Research, 6, 37-55.

Heimberg, R. G. (1994). Cognitive assessment strategies and the measurement of outcome of treatment for socia phobia. Behaviour Research and Therapy, 32, 269-280.

Heimberg, R. G., Dodge, C. S., Hope, D. A., Kennedy, C. R., Zollo, L., y Becker, R. E. (1990). Cognitive behavioral group treatment of social phobia: Comparison 
to a credible placebo control. Cognitive Therapy and Research, 14, 1-23.

Heimberg, R. G., Holt, C. S., Schneier, F. R., Spitzer, R. L., y Liebowitz, M. R. (1993). The issue of subtypes in the diagnosis of social phobia. Journal of Anxiety Disorders, 7, 249-269.

Heimberg, R. G., Hope, D. A., Rapee, R. M., y Bruch, M. A. (1988). The validity of the Social Avoidance and Distress Scale and the Fear of Negative Evaluation Scale with social phobic patients. Behaviour Research and Therapy, 26, 407-410.

Hofmann, S. G., y DiBartolo, P. M. (2000). An Instrument to Assess Self-Statements During Public Speaking: Scale Development and Preliminary Psychometric Properties. Behavior Therapy, 31, 499-515.

Jackson, D. N. (1966). Personality research form. Goshen, N. Y.: Research Psychologists Press.

Jorquera, M., Baños, R. M., Perpiñá, C., y Botella, C. (2005). La Escala de Estima Corporal BES): Validación en una muestra española. Revista de Psicopatología y Psicología Clínica, 3, 173-192.

Leary, M. R. (1983a). A brief versión of the Fear of Negative Evaluation Scale. Personality and Social Psychology Bulletin, 9, 371-375.

Leary, M. R. (1983b). Understanding social anxiety: Social, personality, and clinical perspectivas. Beverly Hills, CA: Sage.

Leary, M. R. (1983c). Social anxiousness: The construct and its measurement. Journal of Personality Assessment, 47, 66-75.

Liebowitz, M. R. (1987). Social phobia. Modern Problems of Pharmacopsychiatry, 22, 141-173.

Marks, I. M., y Matthews, A. M. (1979). Brief standard self-rating for phobic patients. Behaviour Research and Therapy, 17, 263-267.

Marsh, H. W. (1996). Positive and negative global self-steem: A substantively meaningful distinction or artifactors? Journal of Personality and Social Psychology, 70, 810-819.

Mattick, R. P., y Clarke, (1998). Development and validation of measures of social phobia scrutiny fear and social interaction anxiety. Behaviour Research and Therapy, 36, 455-470.

Méndez, F. J., Inglés, C. J., y Hidalgo, M. D. (1999). Propiedades psicométricas del cuestionario de confianza para hablar en público: estudio con una muestra de alumnos de enseñanzas medias. Psicothema, 1, 65-74.

Meyer, T. J., Miller, M. L., Metzger, R. L., y Borkovec, T. D. (1990). Development and validation of the Penn State Worry Questionnaire. Behaviour Research and Therapy, 28, 487-495.

Miller, R. S. (1995). On the nature of embarrassability: Shyness, social evaluation, and social skill. Journal of Personality, 63, 315-339.
Nuevo, R. (2005). Sensibilidad y especificidad del Cuestionario de Preocupación y Ansiedad para la detección del trastorno de ansiedad generalizada. Revista de Psicopatología y Psicología Clínica, 10, 135-141.

Oei, T. P., Kenna, D., y Evans, L. (1991). The reliability, validity, and utility of the SAD and FNE scales for anxiety disorders patients. Personality and Individual Differences, 12, 111-116.

Paul, G. L. (1966). Insight vs. desensitization in psychotherapy. Standford, CA: Standford University Press.

Rapee, R. M., y Heimberg, R. G. (1997). A cognitive-behavioral model of anxiety in social phobia. Behaviour Research and Therapy, 35, 741-756.

Rapee, R. M., y Lim, L. (1992). Discrepancy between selfand observer ratings of performance in socia phobics. Journal of Abnormal Psychology, 101, 728-731.

Reiss, S., Peterson, R. A., Gursky, D. M., y McNally, R. J. (1986). Anxiety sensivity, anxiety frequency and the prediction of fearfulness. Behaviour Research and Therapy, $24,1-8$.

Rodebaugh, T. L., Woods, C. M., Thissen, D. M., Heimberg, R. G., Chambless, D. L., y Rapee, R. M. (2004). More information from fewer questions: The factor structure and item propierties of the original and Brief Fear of Negative Evaluation Scale. Psychological Assessment, 16, 169-181.

Rosenberg, M. (1965). Society and the Adolescent SelfImage. Princeton: Princeton University Press.

Saluck, R. G., Herbert, J. D., Rheingold, A., Harwell, V., Coppola, S. W., y Crittenden, K. (2000, Noviembre). The Brief Fear of Negative Evaluation Scale: Preleminary psychometric findings. Poster presented at the annual meeting of the Association for the Advancement of Behavior Therapy. New Orleans, LA.

Smith, R. E., y Sarason, I. G. (1975). Social Anxiety and the evaluation of negative interpersonal feedback. Journal of Consulting and Clinical Psychology, 43, 429.

Spielberger, C. D., Gorsuch, R. L., y Lushene, R. (1983). Manual for the state-trait anxiety inventory. Palo Alto, CA: Consulting Psychologists Press. (TEA, 1988).

Taylor, J. (1953). A personality scale of manifest anxiety. Journal of Abnormal and Social Psychology, 48, 285-290.

Turner, S. M., Beidel, D. C., Dancu, C. V., y Stanley, M. A. (1989). An Empirically derived inventory to measure social fears and anxiety: The Social Phobia Anxiety (SPAI). Psychological Assessment, 1, 35-40.

Turner, S. M., McCanna, M., y Beidel, D. C. (1987). Validity of Social Avoidance and Distress and Fear of Negative Evaluation Scales. Behaviour Research and Therapy, 25, 113-115.

Villa, H. (1999). Validación de un instrumento de medida en fobia social: Miedo a la Evaluación Negativa (FNE). Tesis de licenciatura no publicada, Univesidad Jaume I. 
Watson, D., y Friend, R. (1969). Measurement of social evaluative anxiety. Journal of Consulting and Clinical Psychology, 33, 448-457.

Weeks, J. W., Heimberg, R. G., Fresco, D. M., Hart, T.A., Turk, C. L., Schneier, F. R., y Liebowitz, M. R. (2005). Empirical Validation and Psychometric Evaluation of teh Brief Fear of Negative Evaluation Scale in Patients
With Social Anxiety Disorder. Psychological Assessment, 17, 179-190.

Winton, E. C., Clark, D. M., y Edelmann, R. J. (1995). Social Anxiety, fear of negative evaluation and the detection of negative emotion in others. Behaviour Research and Therapy, 33, 193-196.

\section{Anexo I. Escala de Miedo a la Evaluación Negativa versión breve (BFNE)}

\section{(Leary, 1983)}

Nombre

Fecha.

Lea detenidamente cada una de las siguientes frases e indique el grado en que le caracterizan de acuerdo con la siguiente escala:

$$
\begin{aligned}
& 1=\text { NADA característico en mí. } \\
& 2=\text { LIGERAMENTE característico en mí. } \\
& 3=\text { MODERADAMENTE característico en mí. } \\
& 4=\text { MUY característico en mí. } \\
& 5=\text { EXTREMADAMENTE característico en mí. }
\end{aligned}
$$

1. Me preocupa lo que la gente pensará de mí, incluso cuando sé que no tiene importancia.

$\begin{array}{lllll}1 & 2 & 3 & 4 & 5 \\ 1 & 2 & 3 & 4 & 5 \\ 1 & 2 & 3 & 4 & 5 \\ 1 & 2 & 3 & 4 & 5 \\ 1 & 2 & 3 & 4 & 5 \\ 1 & 2 & 3 & 4 & 5 \\ 1 & 2 & 3 & 4 & 5 \\ 1 & 2 & 3 & 4 & 5 \\ 1 & 2 & 3 & 4 & 5 \\ & & & & \\ 1 & 2 & 3 & 4 & 5 \\ 1 & 2 & 3 & 4 & 5\end{array}$

2. No me preocupo incluso cuando sé que la gente se está formando una impresión desfavorable de mí.

3. Frecuentemente temo que otras personas se den cuenta de mis limitaciones.

4. Raramente me preocupo de la impresión que estoy causando en los demás.

5. Temo que los demás no me aprueben.

6. Me da miedo que la gente descubra mis defectos.

7. Las opiniones de los demás sobre mí no me molestan.

8. Cuando estoy hablando con alguien me preocupa lo que pueda estar pensando de mí.

9. Generalmente me preocupo por la impresión que pueda causar.

10. Si sé que alguien me está juzgando, eso tiene poco efecto sobre mí. v

11. A veces pienso que estoy demasiado preocupado por lo que otras personas piensan de mí.

12. A menudo me preocupa decir o hacer cosas equivocadas. 